\title{
Improved wavelet hierarchical threshold filter method for optical coherence tomography image de-noising
}

\author{
Jing Cao*, Pinghe Wang*, Bo $\mathrm{Wu}^{\dagger}$, Guohua Shi*,***, Yan Zhang ${ }^{\S}$, Xiqi Li ${ }^{\uparrow}, \|$, \\ Yudong Zhang ${ }^{\top}, \|$ and Yong Liu* \\ *School of Optoelectronic Information \\ University of Electronic Science and Technology of China \\ No. 4, Section 2, North Jianshe Road \\ Chengdu 610054, P. R. China \\ ${ }^{\dagger}$ College of Optoelectronic Technology \\ Chengdu University of Information Technology \\ Chengdu 610225, P. R. China \\ ${ }^{*}$ Suzhou Institute of Biomedical Engineering and Technology \\ Chinese Academy of Sciences \\ Suzhou 215163, P. R. China \\ ${ }^{\S}$ School of Electronic and Communication Engineering \\ Guiyang University \\ Guiyang 550005, P. R. China \\ IInstitute of Optics and Electronics \\ Chinese Academy of Sciences \\ Chengdu 610209, P. R. China \\ "Chinese Academy of Sciences \\ The Key Laboratory on Adaptive Optics \\ Chengdu 610209, P. R. China \\ **ioe_eye@126.com
}

Received 26 September 2017

Accepted 21 December 2017

Published 2 February 2018

\begin{abstract}
According to the speckle feature in Optical coherence tomography (OCT), images with speckle indicate not only noise but also signals, an improved wavelet hierarchical threshold filter (IWHTF) method is proposed. At first, a modified hierarchical threshold-selected algorithm is used to prevent signals from being removed by assessing suitable thresholds for different noise levels. Then, an improved wavelet threshold function based on two traditional threshold functions is proposed to trade-off between speckle removing and sharpness degradation. The
\end{abstract}

${ }^{* *}$ Corresponding author.

This is an Open Access article published by World Scientific Publishing Company. It is distributed under the terms of the Creative Commons Attribution 4.0 (CC-BY) License. Further distribution of this work is permitted, provided the original work is properly cited. 
de-noising results of an OCT finger skin image shows that the IWHTF method obtains better objective evaluation metrics and visual image quality improvement. When $\alpha=0.2, \beta=5.0$ and $K=1.2$, the improved method can achieve $9.58 \mathrm{~dB}$ improvement in signal-to-noise ratio, with sharpness degraded by $3.81 \%$.

Keywords: Optical coherence tomography; wavelets; speckle.

\section{Introduction}

Optical coherence tomography (OCT) is a promising optical imaging technique widely used in ophthalmology and cardiology, due to its advantages including non-invasive, high speed, high resolution and three-dimensional imaging. ${ }^{1-3}$ The quality of images is vital for OCT. In reality, since OCT is based on low-coherence interferometry, ${ }^{4-6}$ it inevitably brings speckle noise to OCT images. Speckle noise degrades OCT images' quality and makes the details of the image blurry. ${ }^{7}$ For better understanding the speckle noise in OCT, many groups have done significant research on its statistics and properties..$^{8-11}$

Many methods have been proposed to attenuate the speckle noise in OCT images, these methods can be divided into two parts: hardware and software methods. Hardware methods are optical approaches that physically remove speckle noise, including frequency ${ }^{12,13}$ and spatial compounding,${ }^{14-18}$ which can significantly improve signal-to-noise ratio of OCT images. But these methods would increase system complexity, meanwhile decrease imaging speed and spatial resolution. ${ }^{12,16}$ Software approaches based on post-processing filtering techniques, including adaptive Wiener filtering, ${ }^{6}$ curvelet domain filtering, ${ }^{19,20}$ contourlet domain filtering, ${ }^{21}$ Csiszars I-divergence regularization, ${ }^{22}$ interval type II fuzzy system, ${ }^{23,24}$ regularized image restoration based on speckle characteristics, ${ }^{25}$ compressed sensing (CS) reconstruction, ${ }^{26-28}$ sparse reconstruction, ${ }^{29-32}$ and wavelet domain filtering ${ }^{33-37}$ can reduce speckle noise by filtering in different transform domain. Wavelet domain filtering is widely accepted as a promising method in de-noising for OCT images.

The fundamental principle of wavelet threshold algorithm is described as follows: An estimated wavelet threshold is used to decide whether the wavelet coefficients are noise or not. Large amplitude wavelet coefficients are regarded as the carrier of useful information, while small amplitude wavelet coefficients are more likely to be the carrier of noise. ${ }^{36}$ Conventional wavelet de-noising algorithm assumes that the level of noise intensity is the same at different sub-bands, so this algorithm uses a global and constant threshold for different wavelet sub-bands. In fact, the speckle noise pattern is uncorrelated and different in OCT images ${ }^{33}$ and speckle noise can also be the carrier of useful information. ${ }^{38}$ In order to protect useful information from being removed in wavelet filtering, it is important to evaluate suitable thresholds for different wavelet sub-bands. Earlier, hard threshold and soft threshold function used in noise reduction would cause ringing effects ${ }^{39}$ and Gibbs phenomenon ${ }^{21,33}$ due to discontinuity and constant deviation in these two functions. So, it is also important to improve the wavelet threshold function.

In this paper, we propose a modified hierarchical threshold-selected algorithm and an improved wavelet threshold function based on the wavelet threshold de-noising algorithm proposed by Donoho and Johnstone. ${ }^{40}$ The modified hierarchical threshold-selected (MHTS) algorithm can provide different and suitable wavelet thresholds for different wavelet decomposition levels. The improved wavelet threshold function (IWTF) has the ability to balance speckle removal and sharpness degradation. The combination of these two improvements can not only increase the contrast and signal-to-noise ratio (SNR) of OCT images, but also preserve the feature of the structural image.

\section{Theory and Principle}

\subsection{Modified hierarchical threshold- selected (MHTS) algorithm}

The basis theory of wavelet threshold method is described as follows: Because of the low correlation or uncorrelation between the noise and useful signals, wavelet transform makes useful signals distribute in high frequency sub-bands, meanwhile the noise signals distribute in low frequency sub-bands. After wavelet decomposition, useful signals concentrate on larger amplitude wavelet coefficients 
and noise signals concentrate on smaller amplitude wavelet coefficients. ${ }^{36}$ For OCT images, noise intensity is different at different spatial or frequency positions, so the selected wavelet threshold for each decomposition level should also be different. In conventional wavelet threshold de-nosing method, an unchanged global threshold is assessed and then used for each decomposition level. This kind of irrationally selected threshold could cause sharpness degradation and images' vagueness by removing some useful information during the de-noise process. Therefore, it is important to modify the thresholdselected method to achieve suitable threshold for different decomposition levels.

In this paper, a hierarchical threshold is put forward based on the conventional global threshold proposed by Donoho. (As shown in Eqs. (1) and (2).) The conventional global threshold (T) proposed by Donoho is defined as follows:

$$
\begin{gathered}
\sigma=\frac{\operatorname{median}\left(\left|H H_{1}\right|\right)}{0.6745}, \\
T=\sigma \sqrt{2 \ln N},
\end{gathered}
$$

where $\sigma$ is the standard deviation of noisy wavelet coefficient, $\mathrm{HH}_{1}$ is the diagonal high frequency coefficient at the decomposition level $1, N$ is the length of the signal. In practice, at high level of wavelet decomposition, wavelet coefficients of useful signal become larger and the noisy wavelet coefficients become smaller. If the selected global threshold is too small, it would excessively process wavelet coefficients of useful information especially in high wavelet decomposition levels, which could cause sharpness degradation. If the selected global threshold is too large, it would retain some speckle noise in OCT images after the filtering process. In order to trade-off the problem that thresholds are either too large or too small, a hierarchical threshold-selected method (as shown in Eqs. (3) and (4)) is present based on the global threshold method

$$
\begin{gathered}
L_{j}=1+\arctan \frac{j}{10}, \\
T_{j}=K \frac{\operatorname{median}\left(\left|H H_{1}\right|\right)}{0.6754} \frac{\sqrt{2 \ln N_{j}}}{L_{j}},
\end{gathered}
$$

where $j$ is the decomposition level, $T_{j}$ is the threshold at $j$ level, $H H_{j}$ is diagonal high frequency coefficient, $N_{j}$ is the length of $H H_{j}$. Compared to the original threshold selected method, the modified method has two advantages: (1) Divided by $L_{j}$, the value of which is always larger than 1 . With the increasing of level $j, L_{j}$ becomes larger. So, the assessment thresholds $T_{j}$ become smaller, which meets the condition that noisy wavelet coefficients are smaller at higher decomposition levels. (2) An alterable parameter $K$ is used to preserve some useful information from being unnecessarily filtered.

\subsection{Improved wavelet threshold function (IWTF)}

Wavelet threshold function is used to remove the noise mainly from low frequency wavelet coefficients. Based on two conventional threshold functions: hard threshold function and soft threshold function, an improved wavelet threshold function is proposed to remedy the defects, such as ringing effect and Gibbs phenomena, ${ }^{21,33,39}$ of these two conventional functions.

The hard threshold function is shown in Eq. (5) as

$$
\bar{w}_{j, k}= \begin{cases}w_{j, k}, & \text { if }\left|w_{j, k}\right| \geq \mathrm{T}, \\ 0, & \text { if }\left|w_{j, k}\right|<0,\end{cases}
$$

where $\bar{w}_{j, k}$ is the wavelet coefficient after threshold processing, $w_{j, k}$ is the original wavelet coefficient, $j$ is the wavelet decomposition level, $k$ is the spatial coordinate, $T$ is the threshold. The discontinuities at threshold $\pm T$ could cause ringing effect and pseudo-Gibbs phenomena, it would obscure edge details of OCT images. The blue curve in Fig. 1(a) shows the plot of the hard threshold function (assuming $T$ is 40 ).

The soft threshold function is shown in Eq. (6):

$$
\bar{w}_{j, k}= \begin{cases}\operatorname{sgn}\left(w_{j, k}\right)\left(\left|w_{j, k}\right|-T\right), & \text { if }\left|w_{j, k}\right| \geq T \\ 0, & \text { if }\left|w_{j, k}\right|<T\end{cases}
$$

where sgn () is a function: if the wavelet coefficient $w_{j, k}$ is larger than zero, then it will become 1 , otherwise it will become -1 . In soft threshold process, since there is a constant deviation between the original wavelet coefficients and the processed wavelet coefficients, this deviation could decrease contrast and smoothness of OCT images. The green curve in Fig. 1(a) shows the plot of the soft threshold function (assuming $T$ is 40).

In order to overcome the discontinuities and constant deviations coming from two conventional threshold functions, a new improved threshold function is presented based on a curvature function. 


\section{J. Cao et al.}
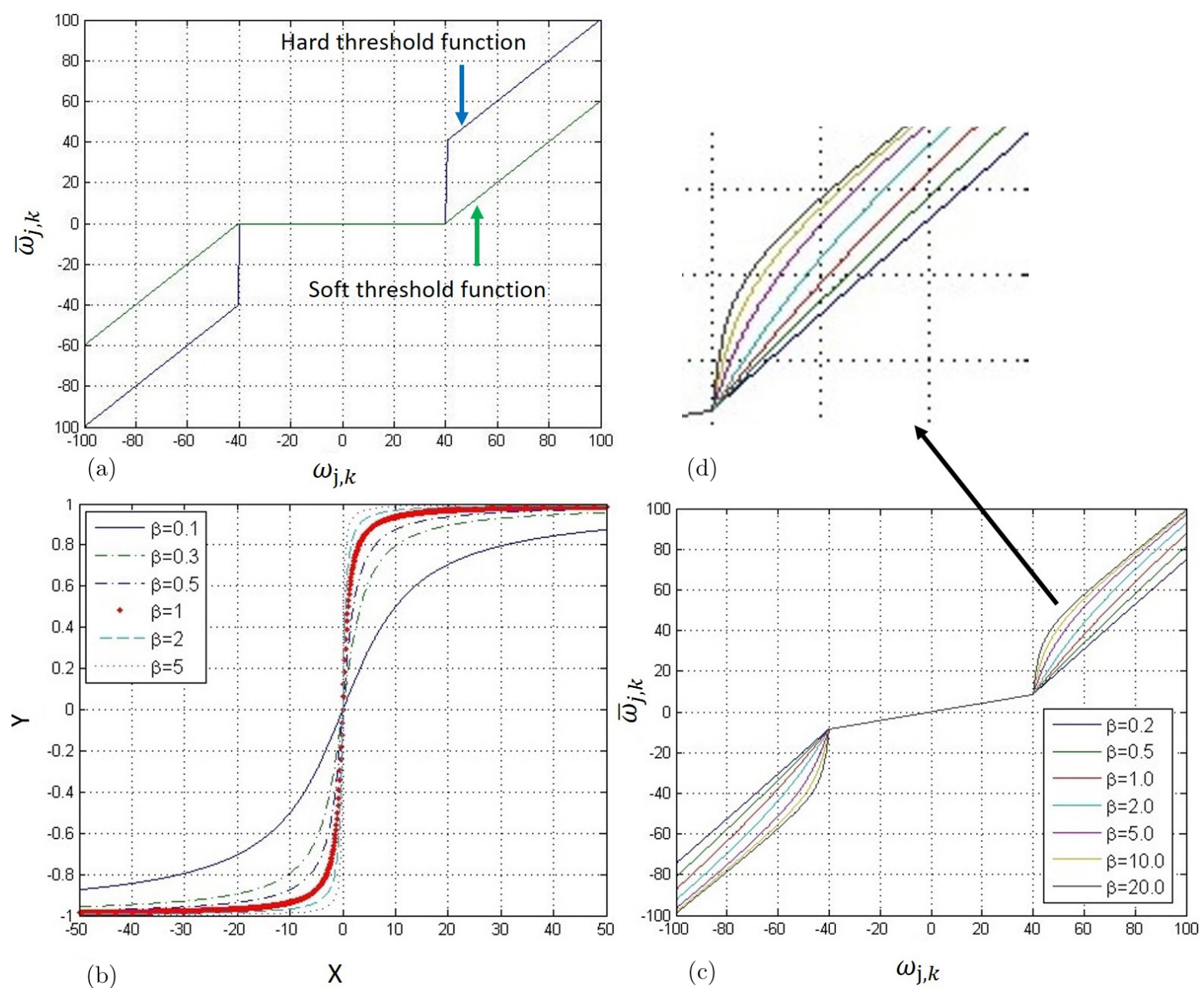

Fig. 1. Diagram of threshold functions. (a) Two conventional threshold functions: Hard (blue curve) and soft (green curve); (b) modified curvature function; (c) the improved threshold function (when $\beta$ is varied from 0.2 to 20); (d) zooming up of the area pointed by black arrow in Fig. 1(c).

The definition of this curvature function is as follows:

$$
y=\frac{2}{\pi} \arctan (\beta x), \beta \in R
$$

The variable $x$ of the function can be any real number on $X$-axis, the variable y can be any real number between $(-1,1)$. This function is zerosymmetric, and the degree of curvature can be adjusted by parameter $\beta$. Figure 1 (b) shows the plot of the modified curvature function when parameter $\beta$ are $0.1,0.3,0.5,1,2$ and 5 , respectively.

Based on the curvature function, a new threshold function is defined as follows

$$
v_{j, k}= \begin{cases}w_{j, k}-T, & \text { if } w_{j, k} \geq T, \\ w_{j, k}+T, & \text { if } w_{j, k} \leq-T,\end{cases}
$$

$$
\bar{w}_{j, k}=\left\{\begin{array}{cc}
v_{j, k}+\frac{2}{\pi} \arctan \left(\beta \frac{v_{j, k}}{T}\right. & \\
\left.+\operatorname{sgn}\left(w_{j, k}\right) \alpha\right) T, & \text { if }\left|w_{j, k}\right| \geq T, \\
\frac{2}{\pi} \arctan (\alpha) w_{j, k}, & \text { if }\left|w_{j, k}\right|<T, \\
0<\alpha<1, \beta>0 &
\end{array}\right.
$$

where parameter $\alpha$ is used to realize different degree of shrinkage on high frequency coefficients. In both hard and soft threshold functions, for those wavelet coefficients, whose absolute value are smaller than $T$, are totally regarded as noise and set to zero. This will inevitably remove some useful information and lead to detail deficiency.

In the new threshold function, the wavelet filtering process would become more reasonable by 
introducing two parameters. For those with $\left|w_{j, k}\right|<T$, the wavelet coefficients after threshold process would be adjusted by choosing an appropriate parameter $\alpha$, rather than setting to zero in previous wavelet functions. For those with $\left|w_{j, k}\right|>T$, the degree of curvature is adjusted by parameter $\beta$. When $\beta$ is 0 , the improved filtering process is equivalent to soft threshold function. When $\beta \rightarrow \infty$, the improved filtering process is equivalent to hard threshold function. Figure 1(c) shows the diagram of improved threshold function at different degrees of curvature, parameter $\beta$ is $0.2,0.5,1.0,2.0,5.0,10.0$ and 20.0 , respectively (assuming threshold $T=40$, parameter $\alpha$ is 0.2 ). Figure 1(d) shows the zooming up of the area pointed by black arrow in Fig. 1(c).

\subsection{Procedure of improved wavelet hierarchical threshold de-noising}

As shown in Fig. 2, there are five basic procedures of the wavelet-based hierarchical threshold de-noising process.

(1) Logarithmically transform. ${ }^{33-36}$ The speckle noise in OCT images is multiplicative noise. ${ }^{34}$ By taking the logarithmical transform of $f(x, y)$, the nonlinear multiplicative noise could be turned into additive noise. The noisy OCT image $f(x, y)$ is presented by Eq. (10) as

$$
f(x, y)=s(x, y) * n(x, y),
$$

where $s(x, y)$ is useful signal, $n(x, y)$ is noise signal. Equation (11) is the logarithmical transform of original OCT image, presented by
Eq. (10) as:

$$
g(x, y)=\ln s(x, y)+\ln n(x, y) .
$$

(2) Two-dimensional discrete wavelet transform (2-D-DWT). The 2-D-DWT is used to decompose the speckle noise into different levels, which are contained in different amplitude wavelet coefficient $w_{j, k}$ on each layer.

(3) Wavelet thresholding filter. A modified hierarchical threshold-selected algorithm and an improved wavelet threshold function are used to optimize the filter process by adjusting three parameters $\alpha, \beta$ and $K$ from Eqs. (4) and (9).

(4) Two-dimensional inverse discrete wavelet transform (2-D-IDWT). It is used to reconstruct the logarithmic image with processed wavelet coefficient $w_{j, k}$.

(5) Exponential transform. Turning the reconstructed image into the noise-free image.

\subsection{Image quality metrics parameters}

Four metrics, including signal-to-noise ratio (SNR), contrast-to-noise ratio (CNR), equivalent number of looks (ENL) and XCOR, are used to assess the image quality after filtering process. ${ }^{25,33,39}$ Among them, SNR is a global metric for the whole image and calculated in linear scale, while CNR and ENL are calculated as the average of several regions of interest (ROI) of the OCT images and measured in logarithmic scale.

The definition of $\mathrm{SNR}^{33}$ is shown in Eq. (12),

$$
\operatorname{SNR}(d B)=10 \log _{10}\left(\frac{\max \left(I^{2}\right)}{\sigma_{n}^{2}}\right),
$$

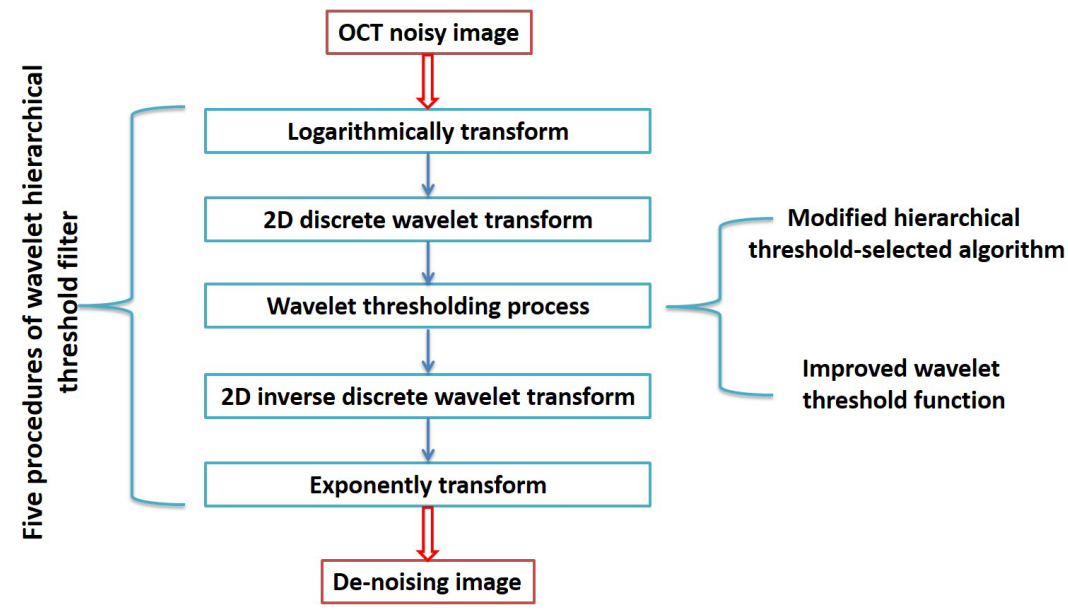

Fig. 2. Procedure of improved wavelet hierarchical threshold de-noising for OCT image. 
where $I$ is the pixel value of the image on linear intensity scale, and $\sigma_{n}$ is the noise standard deviation of the whole image.

The definition of $\mathrm{CNR}^{25}$ is shown in Eq. (13),

$$
\mathrm{CNR}=\frac{1}{R}\left(\sum_{r=1}^{R} \frac{\mu_{r}-\mu_{b}}{\sqrt{\left(\sigma_{r}^{2}+\sigma_{b}^{2}\right)}}\right),
$$

where $R$ is the number of ROIs choosen from the OCT images, $\mu_{r}$ is the mean pixel value and $\sigma_{r}$ is the pixel standard deviation in the $r$ th ROI, $\mu_{b}$ and $\sigma_{b}$ are the mean pixel and standard deviation of the background region of the image, respectively. The CNR indicates the contrast between image feature and an area of background noise.

The definition of $\mathrm{ENL}^{25}$ is shown in Eq. (14),

$$
\mathrm{ENL}=\frac{1}{H}\left(\sum_{h=1}^{H} \frac{\mu_{h}^{2}}{\sigma_{h}^{2}}\right),
$$

where $H$ is the number of ROIs, $\mu_{h}$ is the mean pixel value and $\sigma_{h}$ is the pixel standard deviation in the $h^{\prime}$ th ROI. The ENL indicates smoothness in areas that should have a homogeneous appearance but are corrupted by speckle.

The definition of $\mathrm{XCOR}^{39}$ is shown in Eq. (15),

$$
\mathrm{XCOR}=\frac{\sum_{(i, j)} I_{\text {before }}(i, j) * I_{\mathrm{after}}(i, j)}{\sqrt{\left(\sum_{(i, j)} I_{\text {before }}(i, j)^{2} * \sum_{(i, j)} I_{\mathrm{after}}(i, j)^{2}\right)}},
$$

where $I_{\text {before }}(i, j)$ and $I_{\text {after }}(i, j)$ are the intensity value of each pixel of noisy OCT images and noisefree images, respectively. The value of XCOR is smaller than 1 and will approach 1 when the denoised images resembles the original image.

\section{Experimental Results and Analysis}

In order to demonstrate the effectiveness of the hierarchical threshold-selected method and improved wavelet threshold function, this technique is applied to a human finger skin image from a Swept-source OCT. The image size is $1024 * 656$ pixels. The SSOCT utilizes a broadband swept source (Santec. Inc, HSL-20, $\lambda_{0}=1310 \mathrm{~nm}, \Delta \lambda=107 \mathrm{~nm}$ ) with $12 \mu \mathrm{m}$ axial resolution and $13 \mu \mathrm{m}$ transverse resolutions. The wavelet decomposition level is 3 and processing strategy is the $d b 6$ in Daubechies group. This algorithm is realized in Matlab environment with $320.9 \mathrm{~ms}$ computation time for each 2D image $(1024 * 656$ pixels). The computer we used has an i7 processor. Five regions of interest (ROIs) are marked out (shown in Fig. 6(a)) in original image, that are used to calculate four metrics after the filtering process. Region 1 is background noise region used to estimate noisy level of the whole image. The CNR and ENL are calculated on the average of four ROIs from region 2 to 5 (marked out in the noisy image).

Table 1 shows effect of parameter $K$ in preserving some useful information from being excessively filtered. A $3^{*} 3$ Wiener filter is applied to the image as a comparison. As shown in Table 1, when $K \geq 0.8$, the CNR and ENL of MHTS are significantly larger than that of Wiener filter. The SNR of MHES is larger than that of Wiener filter when $K \geq$ 1.0 and smaller when $K<1.0$. As for the XCOR, the result is another way around. Although the XCOR is decreased $0.15 \%$ to $0.35 \%$, it is still important to get a suitable $K$ in order to prevent some signals from being removed. From Eqs. (3) and (4), it is obvious that when $K<1$, threshold $T$ becomes smaller; when $K>1$, then threshold becomes bigger. By adjusting parameter $K$, meanwhile $\alpha$ and $\beta$ are constant at 0.2 and 5.0, respectively. As shown in Fig. 3(b), the SNR increases maximum at $9.58 \mathrm{~dB}$ with $3.81 \%$ sharpness degradation (when $K$ is 1.2 ) compared to the original image. Meanwhile, from Fig. 3(a), CNR and ENL are also improved with the increase of parameter $K$. It indicates speckles are not always noisy, sometime they could also be the carrier of signals. ${ }^{38}$ Appropriately increasing threshold $T$ could protect these signals from being removed in wavelet filter.

Table 1. Image quality metrics when $K$ varies from 0.7 to $1.3(\alpha=0.2, \beta=5.0)$.

\begin{tabular}{lcccc}
\hline Methods & CNR & ENL & SNR & XCOR \\
\hline Original & 1.94 & 13.21 & 59.54 & 1 \\
Wiener & 3.77 & 36.30 & 67.02 & $96.50 \%$ \\
\hline \multicolumn{5}{c}{ MHTS $(\alpha=0.2, \beta=5.0)$} \\
\hline$K=0.7$ & 3.65 & 35.14 & 65.32 & $96.96 \%$ \\
$K=0.8$ & 4.11 & 42.36 & 65.71 & $96.68 \%$ \\
$K=0.9$ & 4.54 & 49.56 & 66.71 & $96.48 \%$ \\
$K=1.0$ & 4.91 & 55.91 & 67.72 & $96.35 \%$ \\
$K=1.1$ & 5.20 & 60.51 & 68.56 & $96.26 \%$ \\
$K=1.2$ & 5.37 & 63.18 & 69.12 & $96.19 \%$ \\
$K=1.3$ & 5.48 & 64.84 & 68.67 & $96.15 \%$ \\
\hline
\end{tabular}




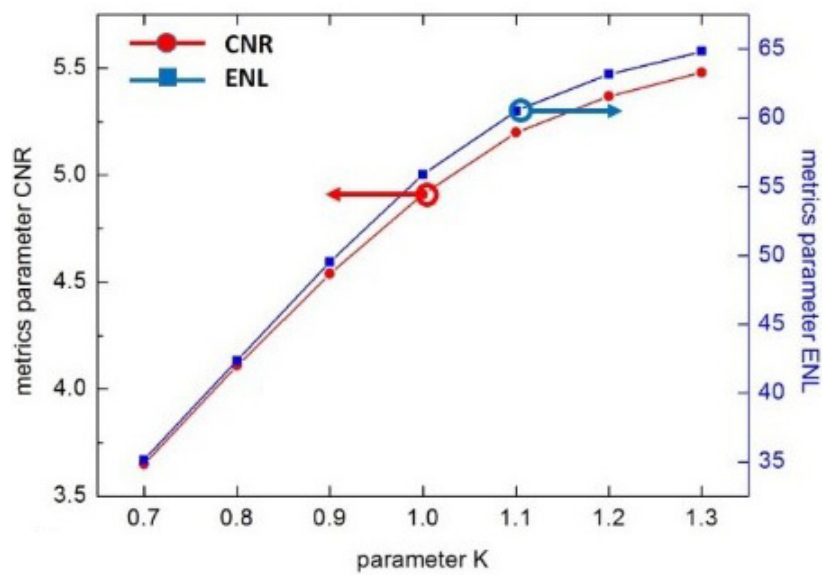

(a)

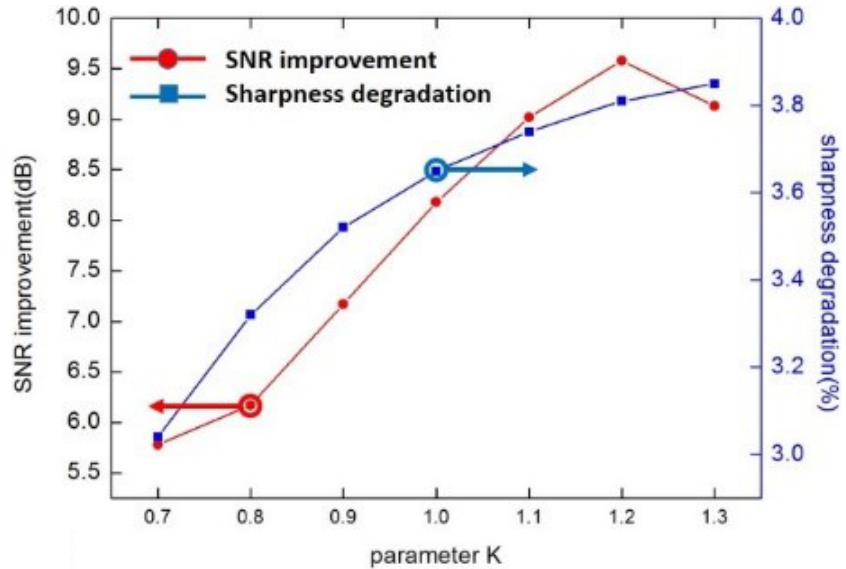

(b)

Fig. 3. Diagram of four metrics vs parameter $K$ (when $\alpha=0.2, \beta=5.0$ ). (a) Calculated CNR and ENL improvement of a SSOCT image when $K$ varies from 0.7 to 1.3. (b) Calculated SNR increasing and sharpness degradation of the image when $K$ varies from 0.7 to 1.3 .

Table 2. Image quality metrics when $\alpha$ varies from 0.0 to $0.5(\beta=5.0, K=1.2)$.

\begin{tabular}{lcccc}
\hline Methods & CNR & ENL & SNR & XCOR \\
\hline \multicolumn{4}{l}{ Conventional wavelet threshold function } & $(K=1.2)$ \\
Hard threshold & 5.19 & 59.87 & 67.71 & $94.86 \%$ \\
Soft threshold & 6.09 & 74.04 & 66.85 & $94.68 \%$ \\
Improved wavelet threshold $(\beta=5.0, K=$ & $1.2)$ \\
$\alpha=0.0$ & 5.81 & 68.58 & 70.56 & $94.81 \%$ \\
$\alpha=0.1$ & 5.67 & 66.84 & 70.07 & $95.54 \%$ \\
$\alpha=0.2$ & 5.37 & 63.18 & 69.12 & $96.19 \%$ \\
$\alpha=0.3$ & 4.99 & 58.29 & 67.96 & $96.77 \%$ \\
$\alpha=0.4$ & 4.62 & 53.07 & 66.79 & $97.26 \%$ \\
$\alpha=0.5$ & 4.27 & 48.11 & 65.69 & $97.68 \%$ \\
\hline
\end{tabular}

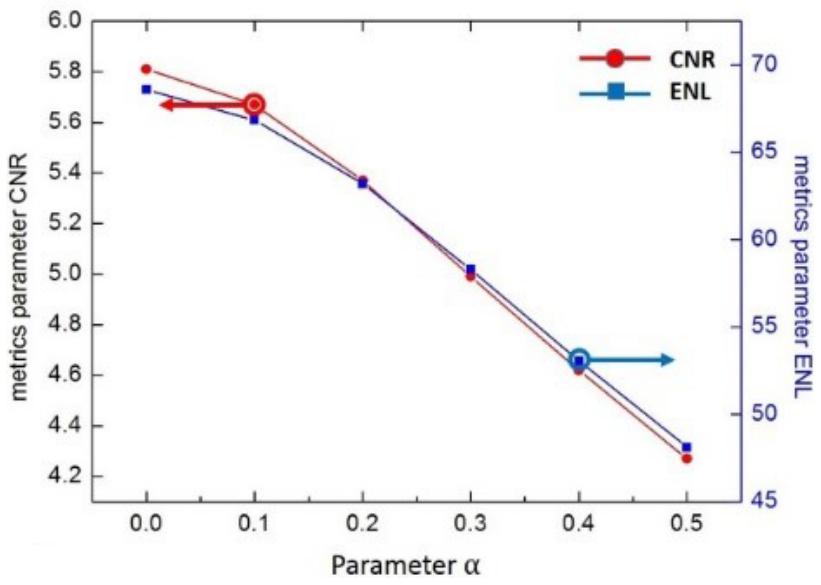

(a)
Table 2 shows effect of parameter $\alpha$ in trade-off between speckle removing and sharpness degradation (when $\beta=5.0, K=1.2$ ). With the decreasing of parameter $\alpha$ from 0.5 to 0.0 , the SNR has improved from $6.15 \mathrm{~dB}$ to $11.02 \mathrm{~dB}$ compared to original image, as well as showing prominent advance in both CNR and ENL (as shown in Fig. 4(a)). But it causes sharpness degradation from $2.32 \%$ to $5.19 \%$. Figure 4(b) shows the SNR improvement and sharpness degradation for an SSOCT image, when $\alpha$ is varied from 0.0 to 0.5 . In order to balance the SNR and XCOR, a suitable parameter $\alpha=0.2$ is selected, the improved wavelet method can achieve

Fig. 4. Diagram of four metrics vs parameter $\alpha$ (when $\beta=5.0, K=1.2$ ). (a) Calculated CNR and ENL improvement of the image when $\alpha$ varies from 0.0 to 0.5. (b) Calculated SNR increasing and sharpness degradation of the image when $\alpha$ varies from 0.0 to 0.5 . 
Table 3. Image quality metrics when $\beta$ varies from 0.1 to $5.0(\alpha=0.2, K=1.2)$.

\begin{tabular}{lcccc}
\hline Methods & CNR & ENL & SNR & XCOR \\
\hline \multicolumn{4}{l}{ Conventional wavelet threshold function } & $(K=1.2)$ \\
Hard threshold & 5.19 & 59.87 & 67.71 & $94.86 \%$ \\
Soft threshold & 6.09 & 74.04 & 66.85 & $94.68 \%$ \\
Improved wavelet threshold $(\alpha=0.2, K=$ & $1.2)$ \\
$\beta=0.1$ & 5.57 & 67.73 & 66.11 & $96.11 \%$ \\
$\beta=0.3$ & 5.56 & 67.44 & 66.69 & $96.12 \%$ \\
$\beta=0.5$ & 5.55 & 67.10 & 67.08 & $96.13 \%$ \\
$\beta=1.0$ & 5.52 & 66.51 & 67.59 & $96.15 \%$ \\
$\beta=2.0$ & 5.48 & 65.43 & 67.87 & $96.17 \%$ \\
$\beta=5.0$ & 5.37 & 63.18 & 69.12 & $96.19 \%$ \\
\hline
\end{tabular}

$9.58 \mathrm{~dB}$ improvement in SNR, with sharpness degraded by $3.81 \%$.

As it is shown in Table 3, when parameter $\beta$ varies from 0.1 to 5.0 , SNR increases from $66.11 \mathrm{~dB}$ to $69.12 \mathrm{~dB}$ and XCOR increases from $96.11 \%$ to 96.19\%. But other two metrics (both CNR and ENL) decrease. This kind of changing trend is more visible from Fig. 5, both SNR and XCOR increase at the cost of CNR and ENL decrease. It shows the ability of parameter $\beta$ in trade-off between the increasing of two metrics - SNR and XCOR, and the decreasing of other two metrics - CNR and ENL.

It can be seen from the three tables that the de-noising results of the improved wavelet method is better than Wiener filter, soft threshold and hard threshold wavelet filter. By adjusting the parameters $\alpha, \beta$ and $K$, the improved threshold method has successfully balanced speckle removal and sharpness degradation, as well as protected the balance between image quality improvement and edge detail information. When $\alpha=0.2, \beta=5.0$ and $K=1.2$, the improved wavelet method can achieve $9.58 \mathrm{~dB}$ improvement in SNR, with sharpness degraded by $3.81 \%$.

Figure 6 shows the comparison of an OCT finger image before and after different filtering processes. Figure 6(a) is the original OCT image. Figure 6(e) is the image after improved threshold wavelet filter (when $\alpha=0.2, \beta=5.0$ and $K=1.2$ ). The noisefree image in Fig. 6(e) shows the ability of the improved wavelet method to reduce speckle noise in OCT images. For comparison, the de-speckled images by Wiener filer, soft-threshold wavelet filter and hard-threshold wavelet filter are also shown in Figs. 6(b)-6(d), respectively. In order to improve the visibility of the wavelet filter effect, the 5th ROI are zoomed up (shown in Figs. 6(f) and 6(g)). Figure 6(f) is the zooming up from the 5 th ROI from the original noisy image. Figure $6(\mathrm{~g})$ is the same region after improved wavelet threshold filter. There are a lot of grains in Fig. 6(f), as a result of the existence of speckle noise. While in Fig. 6(g), the image becomes more smooth due to the speckle reducing. The improved wavelet method is not only better than those three filters in aspect of speckle reducing, but also capable in balance between image quality improvement and edge details preserving.

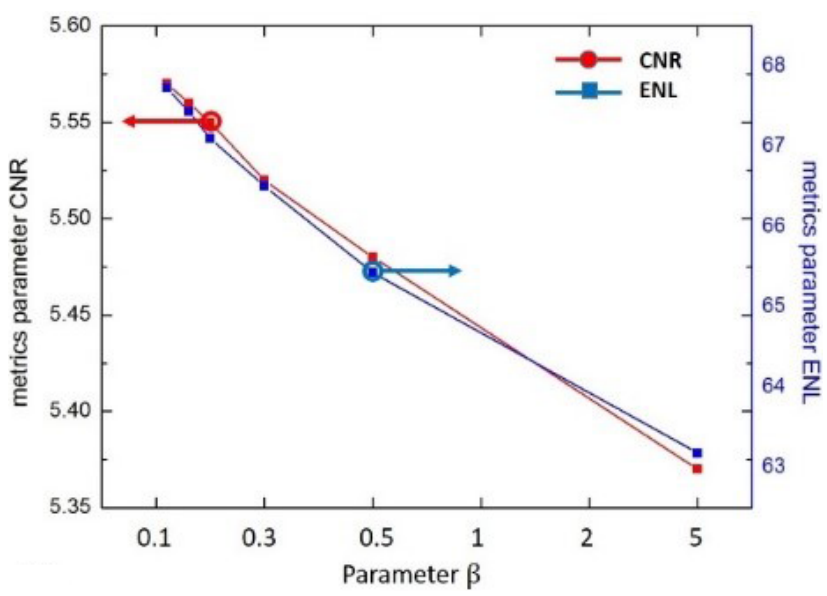

(a)

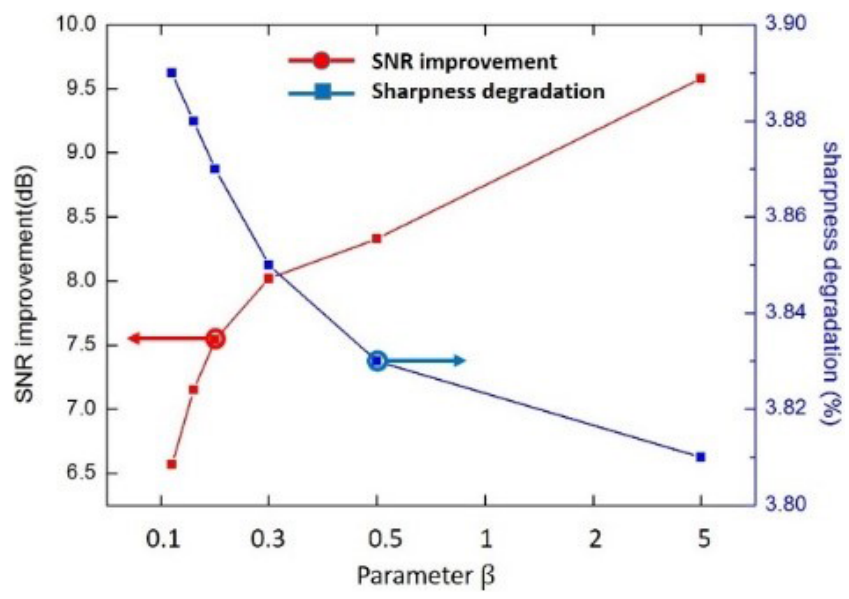

(b)

Fig. 5. Diagram of four metrics vs parameter $\beta$ (when $\alpha=0.2, K=1.2$ ). (a) Calculated CNR and ENL decrease when $\beta$ varies from 0.1 to 5.0. (b) Calculated SNR and XCOR increase when $\beta$ varies from 0.0 to 5.0. 

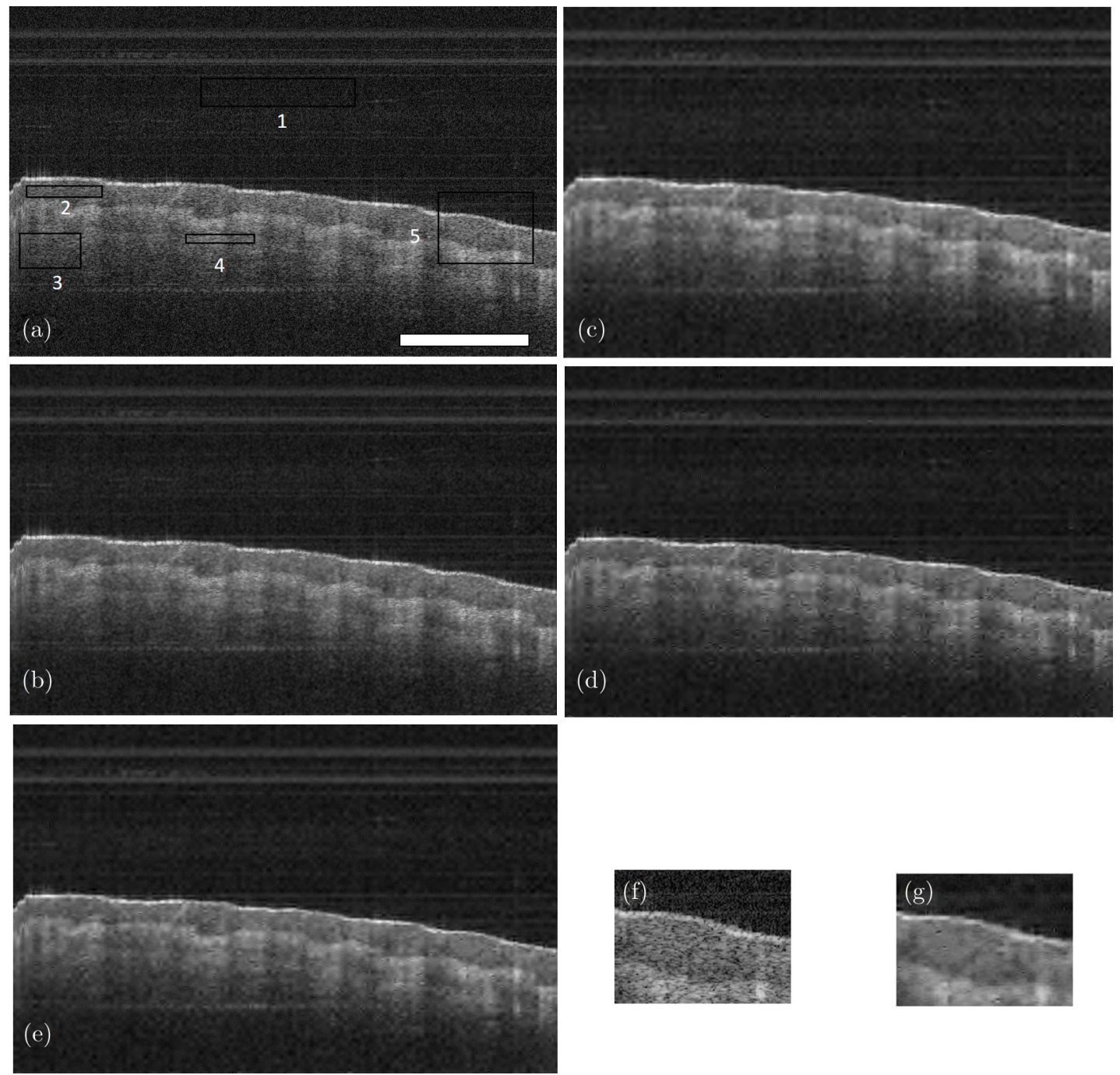

Fig. 6. Comparison of different de-noising methods for an OCT finger skin image. (a) Noisy image with one background region and four ROIs marked in black, (b) image after $3^{*} 3$ Wiener filer, (c) image after soft-threshold wavelet filter, (d) image after hardthreshold wavelet filter, (e) image after improved threshold wavelet filter, (f) zooming up of the 5th ROI from Fig. 6(a), (g) zooming up of the same region from Fig. 6(e). Scale bar in Fig. 6(a) is $500 \mu \mathrm{m}$.

\section{Conclusions}

In OCT systems, the speckle is not only noise but also the carrier of useful information. In this paper, a modified hierarchical threshold-selected algorithm is proposed to protect signals from being removed by adjusting parameter $K$, which is helpful in choosing suitable thresholds for different noise level. An improved wavelet threshold function is used to trade-off between speckle removal and sharpness degradation. This improved threshold function has good continuity and adaptability, parameters $\alpha$ and $\beta$ can be adjusted according to the actual feature of the OCT images. Finally, when $\alpha=0.2, \beta=5.0$ and $K=1.2$, the improved wavelet filter can achieve $9.58 \mathrm{~dB}$ improvement in SNR, with sharpness degraded by $3.81 \%$. The results of OCT images show that the improved threshold filter obtains better objective evaluation metrics and better visual discerniblity.

Except for OCT imaging, wavelet filters have already been applied to some other imaging systems, especially ultrasound imaging. In OCT images, the speckle noise mainly comes from lowcoherence interferometry or the imaging system itself. So, the improved wavelet we present in this paper is suitable for most OCT images/samples and its effectiveness is not limited to images with certain characteristics. 
In this paper, the algorithm is realized in Matlab. It may take more time if we transfer this algorithm to $\mathrm{C}++$ language. But we believe with the help of graphics processing unit (GPU), we can accelerate the filtering process. It can be possible for real-time $2 \mathrm{D}$ or $3 \mathrm{D}$ OCT imaging.

\section{Conflict of Interest}

The authors declare that there are no conflicts of interest related to this paper.

\section{Acknowledgments}

The work is supported by National Nature Science Foundation of China (Nos. 61378090, 61421002, 61505036, 61327004, 61435003 and 61675226); the Sichuan Province International Cooperative Project (No. 2015HH0056); the National Key R\&D Program of China (Nos. 2016YFF0102000, 2016YFF0102003); the Strategic Priority Research Program of the Chinese Academy of Sciences (No. XDB02060000); the Frontier Science Research Project of the Chinese Academy of Sciences (No. QYZDB-SSW-JSC03); the Jiangsu Province Science Fund for Distinguished Young Scholars (No. BK20060010).

\section{References}

1. D. Huang, E. A. Swanson, C. P. Lin, J. S. Schuman, W. G. Stinson, W. Chang, M. R. Hee, T. Flotte, K. Gregory, C. A. Puliafito, J. G. Fujimoto, "Optical coherence tomography," Science $\mathbf{2 5 4}$ (5035), 1178-1181 (1991).

2. E. A. Swanson, J. G. Fujimoto, "The ecosystem that powered the translation of OCT from fundamental research to clinical and commercial impact," Biomed. Opt. Express 8(3), 1638-1664 (2017).

3. S. Huang, M. Shen, D. Zhu, Q. Chen, C. Shi, Z. Chen, F. Lu , "In vivo imaging of retinal hemodynamics with OCT angiography and Doppler OCT," Biomed. Opt. Express 7(2), 663-676 (2016).

4. S. Xiang, L. Zhou, J. Schmitt, "Speckle noise reduction for optical coherence tomography," Proc. SPIE 3196, 7988 (1998).

5. J. M. Schmitt, S. H. Xiang, K. M. Yung, "Speckle in optical coherence tomography," J. Biomed. Opt. 4(1), 95 (1999).

6. A. Ozcan, A. Bilenca, A. E. Desjardins, B. E. Bouma, G. J. Tearney, "Speckle reduction in optical coherence tomography images using digital filtering," J. Opt. Soc. Am. A 24(7), 1901-1910 (2007).

7. M. Bashkansky, J. Reintjes, "Statistics and reduction of speckle in optical coherence tomography," Opt. Lett. 25(8), 545-547 (2000).

8. B. Karamata, K. Hassler, M. Laubscher, T. Lasser, "Speckle statistics in optical coherence tomography." J. Opt. Soc. Am. A 22(4), 593-596 (2005).

9. P. Lee, W. Gao, X. Zhang, "Speckle properties of the logarithmically transformed signal in optical coherence tomography," J. Opt. Soc. Am. A 28(4), 517-522 (2011).

10. M. Li, R. Idoughi, B. Choudhury, W. Heidrich, "Statistical model for OCT image denoising," Biomed. Opt. Express 8, 3903-3917 (2017).

11. K. Yu, L. Ji, L. Wang, P. Xue, "How to optimize OCT image," Opt. Express 9, 24-35 (2001).

12. M. Pircher, E. Gotzinger, R. Leitgeb, A. F. Fercher, C. K. Hitzenberger, "Speckle reduction in optical coherence tomography by frequency compounding," J. Biomed. Opt. 8(3), 565-569 (2003).

13. T. Bajraszewski, M. Wojtkowski, M. Szkulmowski, A. Szkulmowska, R. Huber, A. Kowalczyk, "Improved spectral optical coherence tomography using optical frequency comb," Opt. Express 16(6), 4163-4176 (2008).

14. A. E. Desjardins, B. J. Vakoc, G. J. Tearney, B. E. Bouma, "Speckle reduction in OCT using massivelyparallel detection and frequency-domain ranging," Opt. Express 14(11), 4736-4745 (2006).

15. N. Iftimia, B. E. Bouma, G. J. Tearney, "Speckle reduction in optical coherence tomography by path length encoded angular compounding," J. Biomed. Opt. 8(2), 260-263 (2003).

16. A. E. Desjardins, B. J. Vakoc, W. Y. Oh, S. M. R. Motaghiannezam, G. J. Tearney, B. E. Bouma, "Angle-resolved optical coherence tomography with sequential angular selectivity for speckle reduction," Opt. Express 15(10), 6200-6209 (2007).

17. D. P. Popescu, M. D. Hewko, M. G. Sowa, "Speckle noise attenuation in optical coherence tomography by compounding images acquired at different positions of the sample," Opt. Commun. 269(1), 247251 (2007).

18. P. Li, L. Zhou, Y. Ni, Z. Ding, P. Li, "Angular compounding by full-channel B-scan modulation encoding for optical coherence tomography speckle reduction," J. Biomed. Opt. 21(8), 086014 (2016).

19. Z. Jian, Z. Yu, L. Yu, B. Rao, Z. Chen, B. J. Tromberg, "Speckle attenuation in optical coherence tomography by curvelet shrinkage," Opt. Lett. 34(10), 1516-1518 (2009).

20. Z. Jian, L. Yu, B. Rao, B. J. Tromberg, Z. Chen, "Three-dimensional speckle suppression in optical 
coherence tomography based on the curvelet transform," Opt. Express 18(2), 1024-1032 (2010).

21. J. Xu, H. Ou, E. Y. Lam, P. C. Chui, K. K. Y. Wong, "Speckle reduction of retinal optical coherence tomography based on contourlet shrinkage," Opt. Lett. 38(15), 2900-2903 (2013).

22. D. L. Marks, T. S. Ralston, S. A. Boppart, "Speckle reduction by I-divergence regularization in optical coherence tomography," J. Opt. Soc. Am. A 22(11), 2366-2371 (2005).

23. P. Puvanathasan, K. Bizheva, "Speckle noise reduction algorithm for optical coherence tomography based on interval type II fuzzy set," Opt. Express $\mathbf{1 5}$ (24), 15747-15758 (2007).

24. P. Puvanathasan, K. Bizheva, "Interval type-II fuzzy anisotropic diffusion algorithm for speckle noise reduction in optical coherence tomography images," Opt. Express 17, 733-746 (2009).

25. D. Yin, Y. Gu, P. Xue, "Speckle-constrained variational methods for image restoration in optical coherence tomography," J. Opt. Soc. Am. A 30(5), 878-885 (2013).

26. D. Xu, N. Vaswani, Y. Huang, J. U. Kang, "Modified compressive sensing optical coherence tomography with noise reduction," Opt. Lett. 37, 4209-4211 (2012).

27. X. Liu, J. U. Kang, "Compressive SD-OCT: The application of compressed sensing in spectral domain optical coherence tomography," Opt. Express 18(21), 22010-22019 (2010).

28. N. Zhang, T. Huo, C. Wang, T. Chen, J. Zheng, P. Xue, "Compressed sensing with linear-in-wavenumber sampling in spectral-domain optical coherence tomography," Opt. Lett. 37, 3075-3077 (2012).

29. L. Fang, S. Li, Qi. Nie, J. A. Izatt, C. A. Toth, S. Farsiu, "Sparsity based denoising of spectral domain optical coherence tomography images," Biomed. Opt. Express 3(5), 927-942 (2012).

30. C. Liu, A. Wong, K. Bizheva, P. Fieguth, H. Bie, "Homotopic, non-local sparse reconstruction of optical coherence tomography imagery," Opt. Express 20, 10200-10211 (2012).
31. I. Kopriva, F. Shi, X. Chen, "Enhanced low-rank+ sparsity decomposition for speckle reduction in optical coherence tomography," J. Biomed. Opt. 21 (7), 076008-076008 (2016).

32. L. Fang, S. Li, D. Cunefare, S. Farsiu, "Segmentation based sparse reconstruction of optical coherence tomography images," IEEE Transactions on Medical Imaging 36(2), 407-421 (2017).

33. D. C. Adler, T. H. Ko, J. G. Fujimoto, "Speckle reduction in optical coherence tomography images by use of a spatially adaptive wavelet filter," Opt. Lett. 29(24), 2878-2880 (2004).

34. M. Nasri, H. Nezamabadi-pour, "Image denoising in the wavelet domain using a new adaptive thresholding function," Neurocomputing 72(4), 1012-1025 (2009).

35. J. Xu, H. Ou, C. Sun, P. Chui, V. X. D. Yang, E. Y. Lam, K. K. Y. Wong, "Wavelet domain compounding for speckle reduction in optical coherence tomography," J. Biomed. Opt. 18(9), 096002 (2013).

36. F. Zaki, Y. Wang, H. Su, X. Yuan, X. Liu, "Noise adaptive wavelet thresholding for speckle noise removal in optical coherence tomography," Biomed. Opt. Express 8(5), 2720-2731 (2017).

37. M. A. Mayer, A. Borsdorf, M. Wagner, J. Hornegger, C. Y. Mardin, R. P. Tornow, "Wavelet denoising of multiframe optical coherence tomography data," Biomed. Opt. Express 3, 572-589 (2012).

38. X. Liu, J. C. R. Roman, Y. Huang, Y. Guo, J. U. Kang, "Robust spectral-domain optical coherence tomography speckle model and its cross-correlation coefficient analysis," J. Opt. Soc. Am. A 30(1), 5159 (2013).

39. Y. Du, G. Liu, G. Feng, Z. Chen, "Speckle reduction in optical coherence tomography images based on wave atoms," J. Biomed. Opt. 19(5), 056009 (2014).

40. D. L. Donoho, I. M. Johnstone, "Adapting to unknown smoothness via wavelet shrinkage," J. Ame. Stat. Assoc. 90(432), 1200-1224 (1995). 